\title{
Flexible polyimide microelectrode array for in vivo recordings and current source density analysis
}

\author{
Karen C. Cheung ${ }^{\mathrm{a}, *}$, Philippe Renaud ${ }^{\mathrm{b}}$, Heikki Tanila ${ }^{\mathrm{c}}$, Kaj Djupsund ${ }^{\mathrm{c}}$ \\ ${ }^{a}$ University of British Columbia, Department of Electrical and Computer Engineering, Vancouver, Canada \\ ${ }^{\mathrm{b}}$ Ecole Polytechnique Fédérale de Lausanne, Laboratoire de Microsystèmes, Lausanne, Switzerland \\ ${ }^{\mathrm{c}}$ University of Kuopio, Department of Neurosciences and Neurology, Kuopio, Finland \\ Received 17 March 2006; received in revised form 20 July 2006; accepted 23 August 2006 \\ Available online 5 October 2006
}

\begin{abstract}
This work presents implantable, flexible polymer-based probes with embedded microelectrodes for acute and chronic neural recordings in vivo, as tested on rodents. Acute recordings using this array were done in mice under urethane anesthesia and compared to those made using siliconbased probes manufactured at the Center for Neural Communication Technology, University of Michigan. The two electrode arrays yielded similar results. Recordings with chronically implanted polymer-based electrodes were performed for 60 days post-surgically in awake, behaving rats. The microelectrodes were used to monitor local field potentials and capture laminar differences in function of cortex and hippocampus, and produced response waveforms of undiminished amplitude and signal-to-noise ratios 8 weeks after chronic implantation. The polymer-based electrodes could also be connected to a lesion current to mark specific locations in the tissue. Current source density (CSD) analysis from the recordings depicted a source-sink-composition. Tissue response was assessed 8 weeks after insertion by immunochemical labeling with glial fibrillary acidic protein (GFAP) to identify astrocytes, and histological analysis showed minimal tissue reaction to the implanted structures.
\end{abstract}

(C) 2006 Elsevier B.V. All rights reserved.

Keywords: Flexible microelectrode array; In vivo recording; Laminar analysis; Current source density

\section{Introduction}

Implantable microelectrodes can be used to record neuronal action potentials or local field potentials from within the brain. These signals are in the micro- to millivolt range when recording (single) spike activity or local field potentials as intracortical signals. Implantable multielectrode arrays offer unique insight into brain function. Single-cell recordings give information about neural coding and tuning, while ensemble recordings show how individual neurons interact as coherent assemblies.

The first implantable electrodes to record chronically from the brain were made from insulated microwires. These wire electrodes were developed in the 1950s and were made from tungsten wire, insulated except for the tip. Strumwasser used single 80- $\mu \mathrm{m}$ diameter stainless steel microwires to record from unanesthetized, freely moving animals (Strumwasser, 1958). These recordings lasted for a week or more. This breakthrough

\footnotetext{
* Corresponding author. Tel.: +1 6048274114 .

E-mail address: kcheung@ece.ubc.ca (K.C. Cheung).
}

technique allowed neurophysiologists to focus on the individual neuron and study activity in awake, behaving animals.

The development of photolithographic techniques and silicon etching technology provided a new tool set for fabricating recording electrodes. Silicon probes capable of recording simultaneously from many points were made using standard planar photolithographic CMOS-compatible techniques on silicon wafers. Silicon-based implantable microelectrode arrays have long been used for parallel recording of multiple neuronal elements (Campbell et al., 1991; Wise et al., 2004; Bai and Wise, 2001; Csicsvari et al., 2003). Micromachined silicon arrays present several advantages over traditional micro wire electrodes: the recording site configuration is easily and precisely defined using photolithography; only one microdrive is required to position the array in the tissue; on-chip integrated circuitry can be integrated to reduce cross-talk and noise. However, the mechanical mismatch between the stiff, brittle probe and the soft biological tissue is thought to aggravate inflammation at the implantation site. Micromotion, due to long-term instabilities or rhythmic pulsation of the tissue, can contribute to shear-induced inflammation (Zhong et al., 2001), encouraging the formation 
of a glial scar, which can encapsulate or break the probe with time. This fibrous tissue may isolate the probe electrically from the surrounding neural tissue. The introduction of a flexible probe may thus reduce these side effects.

Polyimide provides good biocompatibility, insulation resistance and dielectric strength in addition to high mechanical flexibility (Richardson et al., 1993; Seo et al., 2004). Polyimides have previously been used in the field of bio-interfaces in such applications as cuff electrodes and sieve electrodes (Rodriguez et al., 2000; Stieglitz et al., 2002, 2000). Cuff electrodes, which wrap around a nerve trunk, can be used to record sensory signals from peripheral nerves or stimulate motor fibers to activate muscles. Sieve electrodes, which are a thin planar structure perforated by holes, can be placed between the severed ends of the nerve. The regenerated nerve will hold the implant in place, creating an interface between the microelectrode sites near the holes and the nerve. The fabrication method used in this work incorporates several simplifications over previously reported methods (Rousche et al., 2001): the release process is in a saline solution as opposed to a buffered HF bath; by using a dry-etch polyimide, only one lithography layer is required to pattern the structure outline as a last step compared to the separate lithography steps for each photosensitive polyimide layer. In addition, Pt electrodes are more stable over time than Au electrodes for stimulation, since gold electrodes quickly dissolve when a high potential is applied (Hung, 2003).

With precise inter-electrode distances defined by photolithography, microfabricated arrays can give an accurate depth profile of electrical activity in the tissue. In addition, simultaneously acquired readings from the sensor array can be used for the analysis of electrical function of the laminar structure, based on the local potential properties. Current source density (CSD) is related to the second spatial derivative of the potential, and subsequent CSD analysis can, given the initial assumptions of homogenous tissue conductivity and structure curvature, depict the active electrical sources and sinks responsible for the recorded potentials.

Previous work has focused on the use of silicon microelectrodes in acute electrophysiological recordings. Microelectrodes were used to monitor field potentials, and CSD analysis demonstrated layer-by-layer current sources and sinks in the hippocampal cortex (Townsend et al., 2002; Wu and Leung, 2003, 2001; Kloosterman et al., 2001). In this work, we present multipleuse flexible polymer microelectrodes as an improved tool for multi-channel recordings. We show examples of acute as well as chronic recordings, used to capture the laminar differences in function of cortex and hippocampus. This work also presents preliminary examination of the tissue reaction to these implanted polymer-based arrays.

\section{Materials and methods}

The polyimide microarrays consist of alternating layers of polyimide-platinum-polyimide, which are patterned using reactive ion etching (Metz et al., 2004a,b). The microarrays were structured on a solid substrate and are released following the anodic dissolution of a sacrificial aluminum layer under- neath the polyimide (Metz et al., 2005). Electrode implantation and recordings were done at the University of Kuopio. All experiments were conducted in accordance with the Council of Europe guidelines and approved by the Institutional Animal Care and Use Committee and the State Provincial Office of Eastern Finland.

\subsection{Array microfabrication}

Chrome $(200 \mathrm{~nm})$ and aluminum $(500 \mathrm{~nm})$ were evaporated onto a carrier silicon wafer. A $20-\mu \mathrm{m}$ layer of polyimide precursor (PI-2611, HD Microsystems) was applied on top of the aluminum by spin-coating and cured at $300^{\circ} \mathrm{C}$ for $1 \mathrm{~h}$ in a nitrogen atmosphere. A titanium adhesion layer $(50 \mathrm{~nm})$ and platinum layer $(200 \mathrm{~nm})$ were then sputtered onto the cured polyimide. Electrodes were patterned by reactive ion etching in $\mathrm{Cl}_{2}$ using patterned photoresist as an etch mask. A second layer of polyimide (1.5 $\mu \mathrm{m}$ thickness, PI-2610, HD Microsystems), which separates the two electrode layers, was spun on and likewise cured. A second Ti/Pt metal layer was sputtered and patterned to create a second layer of electrodes. The third and final layer of polyimide, which insulates the electrode lines, was spun on and cured. An etch mask of sputtered $\mathrm{SiO}_{2}(500 \mathrm{~nm})$ was deposited onto the wafer and then patterned by reactive ion etching using a photoresist etch mask. This oxide layer was then used as the etch mask during the subsequent oxygen-plasma etch of the polyimide. This final etch defines both the structure outline and opens contacts to the electrodes. The polyimide devices were then detached from the silicon carrier wafer by anodic metal dissolution in a $10 \mathrm{wt} \%$ sodium chloride solution: the substrates were immersed in the salt solution at room temperature with a platinum counter electrode, and a constant positive potential $(0.7 \mathrm{~V})$ was applied to the aluminum layer. The anodic metal dissolution process dissolved the aluminum, leaving the chrome on the substrate, thus releasing the polyimide structures (Fig. 1).

A surface-mount connector (Samtec, Inc.) was soldered onto the electrode bond pads at the end of each polyimide structure and the electrical contacts were insulated with epoxy. This 16channel connector provided the contact between the polyimide structures and the Neuralynx preamplifier.

\subsection{Common recording equipment}

During recordings, the signal from the recording electrodes was first passed through a preamplifier (Neuralynx Inc.) mounted on the distal end of the recording cable and into the main amplifier (Grass Instruments) and the data acquisition PC (running DataWave SciWorks). The auditory stimuli were pure $3.3 \mathrm{kHz}, 80 \mathrm{~dB}$ beeps of $10 \mathrm{~ms}$ duration, generated by a piezoelectric buzzer. The stimuli consisted either of paired pulse beeps (gating paradigm) or of sets of 10 pulses with pulse intervals of $500 \mathrm{~ms}$, followed by a $3.5 \mathrm{~s}$ silence. The trials were averaged in sets of 50 .

\subsection{Acute recording details}

FVB/N mice weighing $25-35 \mathrm{~g}$ were anesthetized with $1.2-1.5 \mathrm{~g} / \mathrm{kg}$ urethane intraperitoneally and placed in a stereo- 
2.
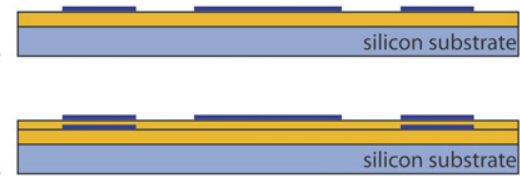

4.
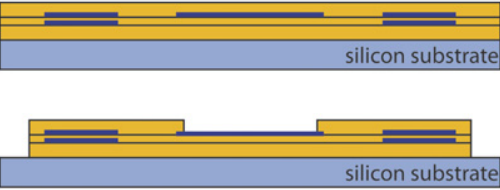

6

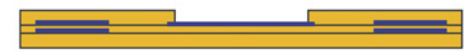

$\mathrm{Cr} / \mathrm{A}$

The first polyimide layer is spun onto the silicon handle wafer, which has a $\mathrm{Cr} / \mathrm{Al}$ sacrifical layer.

$\mathrm{Ti}(50 \mathrm{~nm})$ and Pt (200 nm) electrodes are sputtered and patterned in RIE.

A second layer of polyimide is spun onto the wafer, and a second layer of Ti/Pt electrodes are patterned.

The third and final layer of polyimide is spun onto the wafer.

An oxygen plasma etch opens electrode contacts and defines the probe outlines.

The probes are detached in an anodic release process.

Fig. 1. Cross-sectional view of the microfabrication process. The microelectrodes consist of alternating layers of polyimide-platinum-polyimide, which are patterned using reactive ion etching (Metz et al., 2005).

taxic apparatus (Narishige Instruments). Animal body temperature was maintained at $35 \pm 1{ }^{\circ} \mathrm{C}$ using a custom-made water bed. Holes for the recording and surface (reference) electrodes were drilled on the same side of the animal. The ground electrode (screw) was placed at A1, L1 with respect to bregma. The multielectrode array was inserted vertically at P2.2, L1.6-2.2, into post-parietal cortex and hippocampus.

The multielectrode array was connected to a custom-made printed circuit board with a $2 \times 8$ integrated circuit plug, fitting into a matching plug containing the preamplifier block and a separate ground wire to the reference. At the end of the first recording series, the polyimide microelectrode array was replaced with a microfabricated 16-sensor one-shank silicon multielectrode array (Center for Neural Communication Technology, University of Michigan). A second recording series was performed.

The animal was sacrificed at the end of the experiment with an overdose of injected urethane and incinerated.

\subsection{Chronic recording details}

Male Sprague-Dawley rats were obtained from the National Public Health Institute in Kuopio. They were anesthetized $(30 \mathrm{mg} / \mathrm{kg}$ i.p. pentobarbitol and chloral hydrate, with supportive doses of ketamine $20 \mathrm{mg} / \mathrm{kg}$ i.m.). After drilling, the multielectrode array was implanted vertically into post-parietal cortex and hippocampus. In addition, four reference screw electrodes, wired to a ground connector with plugs for each screw, were attached to the frontal and parietal parts of the skull. The electrode connector, the screws, and their connector were attached to the skull by acrylic cement, which formed a block on the top of the head. After surgery the animals were allowed to heal for at least 1 week before starting a recording session.

\subsection{Electrode marking and tissue preparation}

After acute recordings were finished, a current was led through the microelectrode array to mark the sensor positions.
A $30 \mu \mathrm{A}$ positive current was led for $10 \mathrm{~s}$ through the outermost sensors. The animals were sacrificed by cardiac perfusion, at first with saline and then $4 \%$ paraformaldehyde. The brain was then removed and kept in $4 \%$ paraformaldehyde for 2 days, then sliced into $60-\mu \mathrm{m}$ thick sections using a microtome. The brain was dissected for verification of the anatomy and for experimental precision, and the local electrolytic lesions were observed in postmortem histology. In order to assess the tissue reaction and glial scar formation around chronic implants (Turner et al., 1999), the tissue was stained using anti-glial fibrillary acidic protein (GFAP, Sigma-Aldrich Finland). The number of cells stained was counted around the area of the electrode tested in the chronic experiment.

\subsection{Current source density analysis}

Transmembrane currents flow across the membrane of active neurons and create an ensemble of current sources and sinks: sources inject current into the extracellular medium and sinks absorb current from the medium into a neuronal element (Nicholson and Freeman, 1975). Current source density analysis is used to yield the amplitude of a current source or sink at a given layer in the tissue. The use of CSD gives a better spatial resolution than the original field potential information (Mitzdorf, 1985), removes components created by volume conduction in tissue (Leung, 1990), and provides a better indication of neuronal function. Field potential profiles, in our case the laminar profiles of the auditory-evoked potentials, were measured at equidistant locations ( $100 \mu \mathrm{m}$ spacing between sensors along the probe shank). The one-dimensional CSD $I_{m}$ as a function of depth $z$ and time $t$ was calculated according to a finite difference formula for the second spatial derivative:

$\frac{\partial^{2} \varphi}{\partial^{2} z} \approx \frac{\varphi(z+n \cdot \Delta z)-2 \varphi(z)+\varphi(z-n \cdot \Delta z)}{(n \cdot \Delta z)^{2}}$

$\sigma_{z} \cdot \frac{\partial^{2} \varphi}{\partial^{2} z}=-I_{m}$ 
(a)
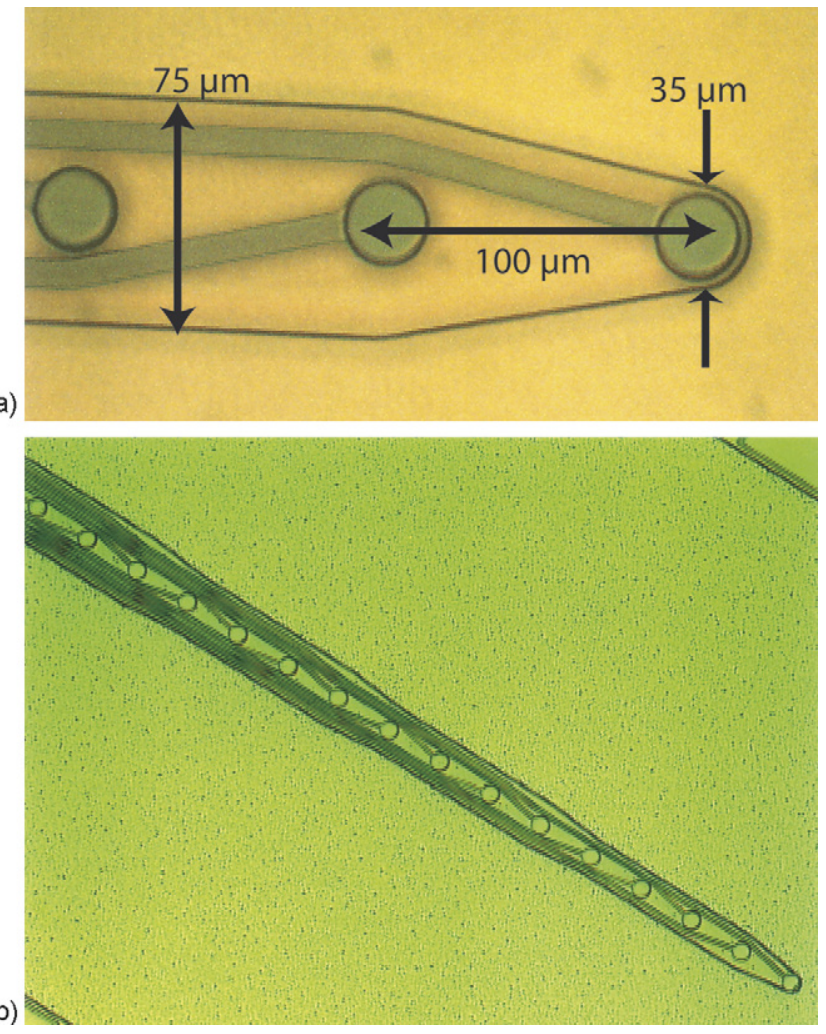

Fig. 2. Micrographs of the microfabricated structures. The probe thickness is $15 \mu \mathrm{m}$. Each electrode is a circle of $25 \mu \mathrm{m}$ diameter. The polyimide is transparent and the overlapping stacked metal lines are visible.

where $\varphi$ is the potential at depth $z, \Delta z$ the spacing between adjacent recording sites, $100 \mu \mathrm{m}$ and $\sigma_{z}$ is conductivity in the $z$ direction. The parameter $n$ defines the amount of spatial smoothing applied to the data (Freeman and Nicholson, 1975); we used $n=1$ throughout (Kloosterman et al., 2004). The parameter $\sigma_{z}$ was assumed to be constant and the CSDs were calculated in arbitrary units $\left(\mathrm{mV} / \mathrm{mm}^{2}\right)$ proportional to the actual current densities. The currents in the other directions were assumed to be negligible (Leung, 1979).

\section{Results and discussion}

\subsection{Microfabrication}

The polyimide microdevices can be re-used several times despite being bent into acute angles. This robustness, an advantage for in vivo applications, contrasts with the brittleness of silicon-based microelectrodes. By stacking the metal layers, the number of electrode sites can be increased without increasing the width of the implanted structure, which will help to minimize damage to the tissue during insertion. The width of the shank increased from $35 \mu \mathrm{m}$ at the tip to $195 \mu \mathrm{m}$ at the farthest recording site from the tip. The shank thickness was $15 \mu \mathrm{m}$ (Fig. 2). Despite their flexibility, the polymer microdevices can be inserted into the tissue without using a guide. The single polyimide structure comprises the recording tip and the flexcable, which traverses the skull and connects the tip to the bond pads for further connection (Fig. 3). Thus, these microfabri-

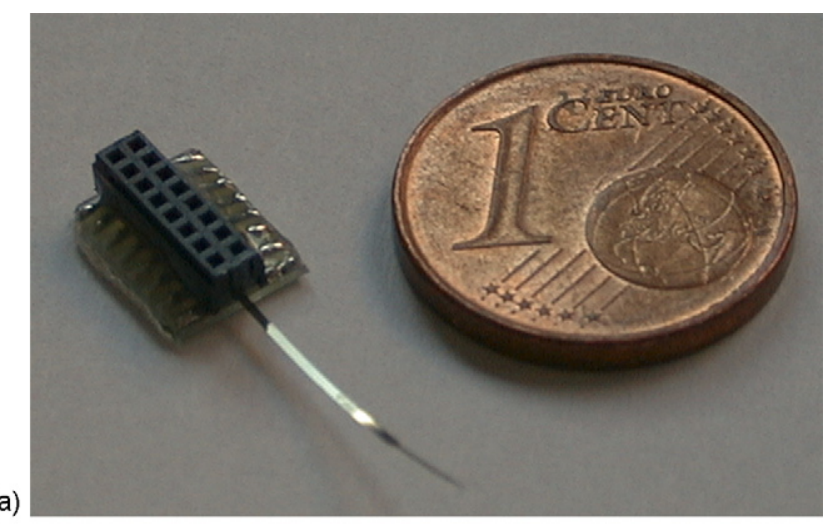

(a)

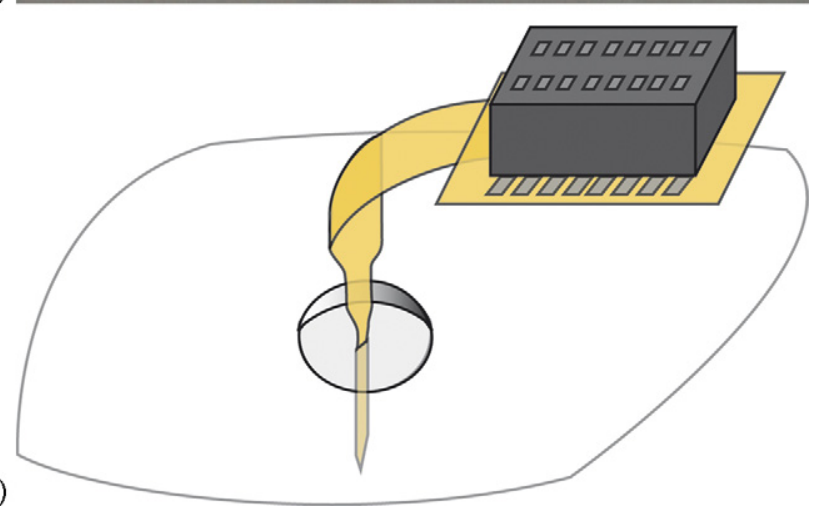

Fig. 3. (a) A micro surface-mount connector is soldered to the polyimide probe. (b) During recording, the microelectrodes are connected to a head-mounted preamplifier, which is mounted on the distal end of the recording cable and main amplifier.

cated structures require minimal packaging when compared to the silicon-based probes.

The electrodes were immersed in phosphate buffered saline (PBS, Sigma, USA; NaCl $120 \mathrm{mM}, \mathrm{KCl} 2.7 \mathrm{mM}$, phosphate buffer $10 \mathrm{mM}, \mathrm{pH} 7.4$ ) and characterized by impedance spectroscopy (Agilent 4292A) from $100 \mathrm{~Hz}$ to $1 \mathrm{MHz}$. The impedance of each sensor, which was a $25-\mu \mathrm{m}$ diameter circle (surface area was $491 \mu \mathrm{m}^{2}$ ) was approximately $1 \mathrm{M} \Omega$ at $1 \mathrm{kHz}$.

Since platinum has good resistance to chemical corrosion, low threshold potential and good biocompatibility, it has often been used as an electrode material for biological applications. Sputter deposition of thin platinum films is also a widely used microfabrication process. For electrical stimulation using biphasic pulsing with optimized parameters, platinum electrode corrosion can be minimized but dissolution may still occur, which would contraindicate the use of thin-film electrodes. Oxidebased electrodes such as iridium do not exhibit corrosion, and in addition have large surface roughness, which contributes to a larger real surface area and lower electrode impedance. The sputtered thin-film Pt electrodes have smooth surfaces and relatively higher impedance. For long-term applications requiring electrical stimulation these thin-film microelectrodes could be platinized to increase thickness and surface roughness while simultaneously decreasing impedance (de Haro et al., 2002), or iridium oxide films could be electrodeposited onto the microfabricated Pt electrodes (Ges et al., 2005). 


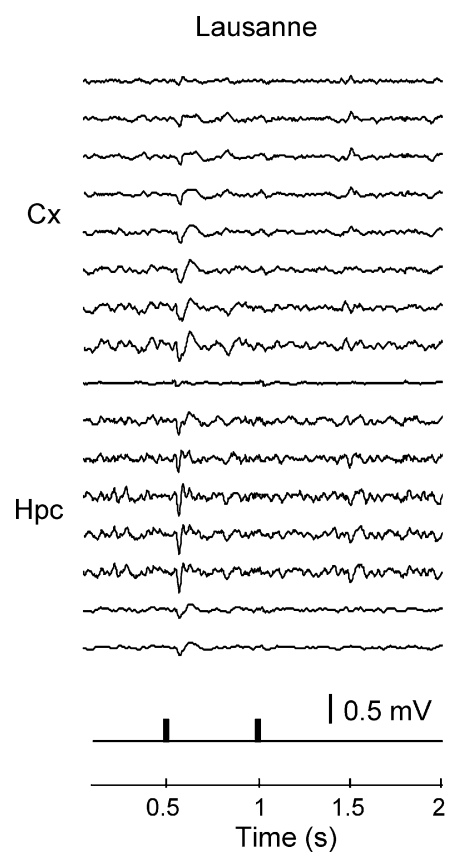

Michigan

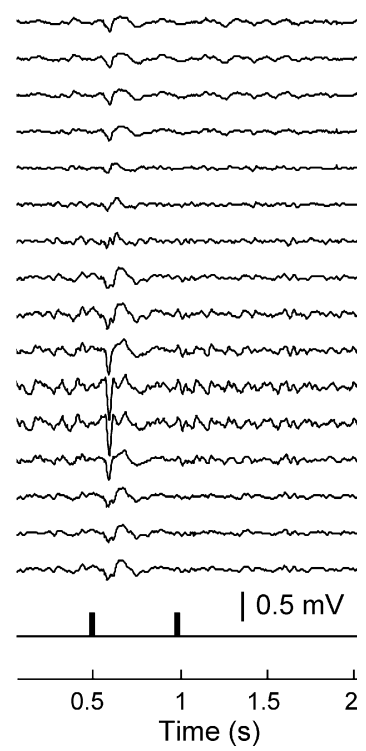

Fig. 4. Laminar profiles of average evoked potentials acquired using the EPFL and Michigan electrodes. Both are linear 16-channel arrays with $100 \mu \mathrm{m}$ spacing between the sensors. Acute measurements in the mouse post-parietal cortex (upper channels) and hippocampus (lower channels). Pairs of sound pulses are shown on the bottom. The vertical scale is in millivolt with the largest signals $\sim 0.7 \mathrm{mV}$.

\subsection{Recording}

During both acute and chronic recording sessions the electrodes yielded stable recordings comparable to those obtained from commercially available silicon electrodes when inserted into cortex and hippocampus, as observed in the form of the standard response parameters, such as latencies, onsets and decays of main components in voltage and CSD traces. During acute recordings (Fig. 4) the responsiveness was only lim- ited by anesthesia stability and reproducible traces could be obtained typically for 4-6h during a recording session. In chronic recordings (Fig. 5) responses of undiminished amplitude could be obtained during 8 weeks, at which point the animal was sacrificed.

The time scale of chronic implants varies and can often be stretched to months. Micro-wire electrodes have been employed to record brain (single-unit) activity since 1958 (Strumwasser, 1958). Over the years, these electrodes have both been found useful and the techniques increased in complexity and sophistication (Lemon, 1973; Buzsaki, 2004). We chose the time scale used as a compromise between practicality and a reasonable time for verification of operation of the polymer-based electrodes.

Fig. 4 shows acute recordings from the mouse, with a 16channel polyimide electrode (left) and a 16-channel commercial silicon electrode (right) going through the post-parietal cortex and the hippocampus. The differences in waveform in deep layers depend on the local structure of hippocampus at the insertion track and minor location differences. The lowest line was the timing of the stimulus: a $10 \mathrm{~ms}$ sound beep pair every $10 \mathrm{~s}$, with an average of 50 trials. The polyimide electrode had a higher noise level given the higher impedance compared to the silicon electrode (a 2/1 ratio for the electrode pair used here), thus also a more local pick-up of signals. Note the silent channel in the polyimide electrode, providing an indication of the intrinsic noise level. The channels are purposely depicted at different heights since the uppermost channel in the polyimide electrode was closer to the surface of the brain. The depth difference of $100 \mu \mathrm{m}$ was estimated from electrolytic markings done at insertion of the polyimide sensors.

Fig. 5 shows the recorded voltage and calculated CSD traces to sets of sound pulses in an experiment using a chronically implanted 16-channel polyimide electrode in the rat. The record is made with a $3-300 \mathrm{~Hz}$ bandpass filter, with a frontal lobe screw serving as the reference. The trace set reveals several current sinks and sources.

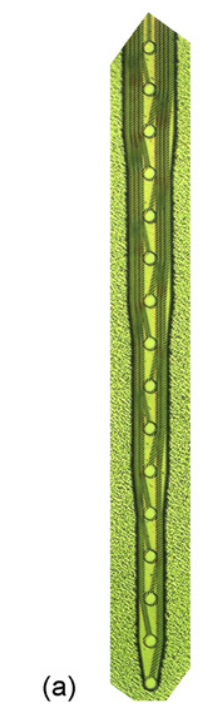

(b)
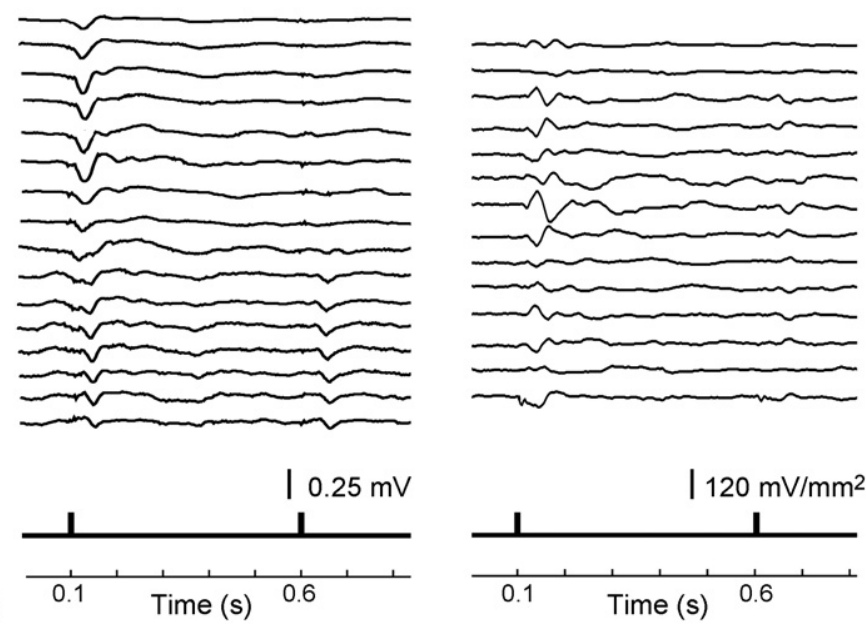

Fig. 5. (a) The 16-channel multielectrode array. (b). Recorded voltage (left, $\mathrm{mV}$ ) and calculated CSD traces (right, $\mathrm{mV} / \mathrm{mm}^{2}$ ) to sets of sound pulses in an experiment using a chronically implanted 16-channel polyimide electrode. The surface of the brain (above the post-parietal cortex) is up and the hippocampus is towards the bottom. The timing of sound pulses is depicted below the lowest trace. 
The hippocampus receives its major sensory input through the entorhinal cortex, which in turn, receives the signals from various association cortices in the brain (Van Hoesen, 1982; Witter, 1993). In a large number of previously published papers (Leung et al., 1992, 1995; Brankack et al., 1993; Liu and Bilkey, 1997a,b; Canning and Leung, 1997; Bragin et al., 2000; Townsend et al., 2002; Bartesaghi and Gessi, 2003; Kloosterman et al., 2004; Gnatkovsky and de Curtis, 2006) intracranial electrical stimulation to one or several hippocampal and/or cortical pathways, such as the components of the perforant path, the hippocampal commissurae and parts of the hippocampus itself, was applied to yield a set of responses for source localization and CSD analysis. In some cases, this stimulation paradigm has been extended towards the auditory pathways (Canning et al., 2000).

In contrast to electrical stimulation, our auditory beeps represent a relatively natural sensory stimulus, applicable for both acute and chronic experiments. At the recording site the responses have been modified throughout the natural auditory pathway and cortices and can be expected to have a signal strength comparable to those triggered by events in everyday life. Thus, the form and temporal characteristics of the responses elicited by these stimuli may differ considerably from those due to electrical stimulation.

The current markings were readily observable in tissue slices (Fig. 6a), indicating good targeting and no shorts between the sensors at current levels used. These lesions, made by $30 \mu \mathrm{A}$ for $10 \mathrm{~s}$, had an approximate radius of $120 \mu \mathrm{m}$.

\subsection{Tissue reaction}

One of the great challenges to maintaining a stable, longterm neural interface is the biological response to electrodes implanted in the central nervous system. Although current microelectrode arrays perform well in acute applications, chronic performance remains inconsistent. Using microwire arrays, Nicolelis et al. (2003) reported a steady drop in the number of functional electrodes remaining over time, decreasing from $\sim 100$ initially implanted microwires to 421 recorded neurons after 3-4 weeks, ending with 58 neurons after 18 months in one subject. In response to brain injury, astrocytes, microglia and other cells proliferate, forming a glial scar (Fawcett and Asher, 1999). Glial fibrillary acidic protein is a major structural component of astrocytes and is generally accepted as an astrocytespecific marker in the central nervous system. To assess the level of scar formation after implantation, an anti-GFAP stain was used in brain preparations of rats used in the chronic experiments. A cell count around the area of the electrode yielded a non-significant level of GFAP-positive cells along the length of the shank. At the insertion area of the cortex and approximately $1 \mathrm{~mm}$ depth there were $16 \pm 1.4$ (standard error of mean) stained cells $/ 0.01 \mathrm{~mm}^{2}$ but this quickly decreased to a non-observable level of stained cells in the hippocampus (Fig. 6b).

Previous work examining astrocyte response to brain injury has shown there are two components of the reactive response to device insertion into the brain. The early response is observed immediately after device insertion, followed by a long-term sustained response. The early response starts within hours of injury
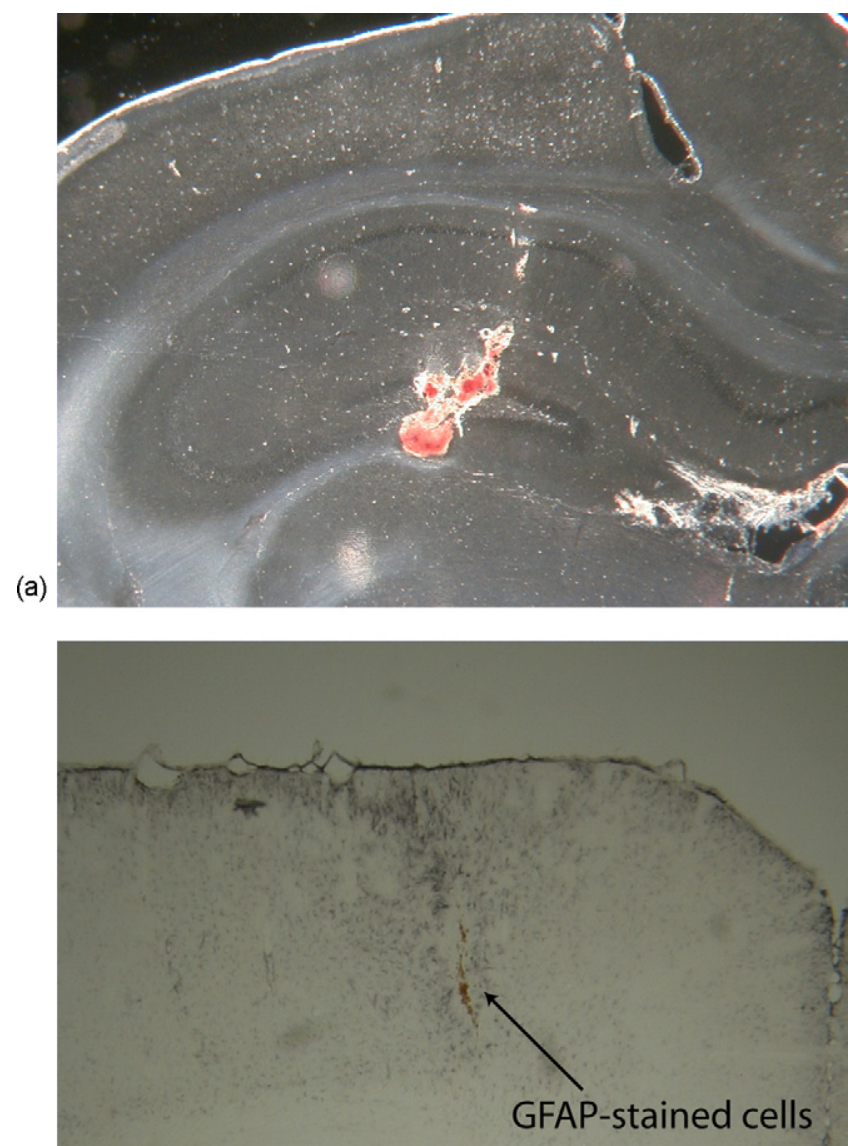

(b)

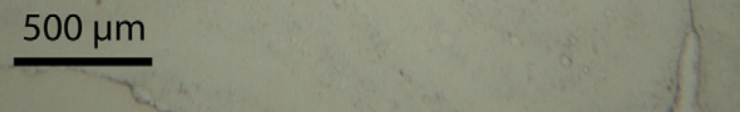

Fig. 6. (a) Current marking in mouse cortex and hippocampus using $30 \mu \mathrm{A}$ during $10 \mathrm{~s}$ after recordings in an acute experiment. (b) Anti-GFAP activity around the implanted cortical area of a rat, 60 days after implantation. No electrolytic lesioning was used for this animal to preserve tissue for cell count. The counts at levels with observable staining, starting from a depth of roughly $1 \mathrm{~mm}$, yielded 16 ( \pm 1.4 S.E.M.) cells $/ 0.01 \mathrm{~mm}^{2}$.

and is characterized by a large number of astrocyte and microglia in the area surrounding the device (Szarowski et al., 2003; Turner et al., 1999). This area can extend to a few hundred microns around the insertion site, and the response is proportional to the cross-sectional area of the devices. This early reaction reaches a peak from 3 to 7 days post-injury (Norton et al., 1992) and then gradually resolves to the long-term response. The longterm chronic response features a compact sheath surrounding the device extending and extending outwards a few tens of microns. This prolonged response has been found to be independent of the device size, geometry and surface roughness. Both Turner et al. and Szarowski et al. have examined the encapsulation 
of micromachined silicon devices of different size and crosssection. Szarowski showed a clear sheath of GFAP-positive astrocytes within a 50-100 $\mu \mathrm{m}$ radius around the insertion site after 4 weeks, and an increased number of GFAP-positive stellate astrocytes to a radius of $500 \mu \mathrm{m}$ from the insertion site. Hampton et al. (2004) have studied the long-term brain response to stab injury using a sterile scalpel to cut a lesion into the cortex. This work showed an increased number of GFAP-positive cells around the lesion $\left(>20\right.$ cells $/ \mathrm{mm}^{2}$ within $100 \mu \mathrm{m}$ of the lesion, 14 days post-lesion). Edell et al. (1992) found more gliosis at the tips of implanted silicon arrays after 6 months of implantation, while Schmidt et al. (1993) found comparable levels of gliosis along the length of the $1.5 \mathrm{~mm}$ arrays.

The astrocyte cell counts obtained here, which were from the surface of the cortex to the depth of $1 \mathrm{~mm}$, are comparable to those from Hampton, whose work concerned mainly the cortex. Notably, however, our results showed non-significant levels of GFAP-positive cells at increased depth and no stained cells in the hippocampus. Shehab et al. (1990) note that the type of fixative (paraformaldehyde, acid alcohol, among others) can affect immunocytochemistry, and that immunoreactivity in white and grey matter differed with the fixative, but they do report the staining of astrocytes in the grey matter of the hippocampus fixed in paraformaldehyde. Although our results indicate minimal tissue reaction to implanted polymer-based microelectrodes, a definitive comparison of the biocompatibility of silicon and polymer-based microelectrodes would require the implantation of structures identical in shape, cross-section and length into the same animal for the same duration.

We expect that the flexible electrode array will have better stability for long-term in vivo recording than a silicon-based array, as the polyimide shank permits probe movement in unison with the brain tissue. Here, micromotion is the relative movement between the implant and the brain tissue. Brain movements or pulsations can be attributed to changes in intracranial pressure due to breathing and the cardiac pulse. For implants, which are tethered to the skull, micromotion can also result from relative movement between the skull and brain. Finite element modeling has shown that flexible substrates such as polyimide can reduce strain at the tip of the probe for tangential tethering force when compared to a stiff probe (Subbaroyan et al., 2005). As silicon has a Young's modulus of $\sim 170 \mathrm{GPa}$, while brain tissue has a Young's modulus of $\sim 3 \mathrm{kPa}$, this reduction can be substantial. Although there are elevated strains at the surface of the brain in this case, optimizing the flexible interconnect could reduce this tangential strain as well.

In the past few years, a lot of excitement has been generated by findings that neural recordings can provide movement-related signals (Nicolelis, 2001). This is partly due to the cortical topographic representation, which implies that sensory modalities are mapped to regions in the sensory cortex, while motor cortex is partitioned into regions that emphasize different movements. Recordings from the motor cortex of awake, behaving monkeys can be used to control computer cursors (Donoghue, 2002). With real-time visual feedback, monkeys were able to make real and virtual arm movements in a three dimensional virtual environment (Taylor et al., 2002). These findings were made possible by multi-channel intracranial recording electrodes. Thus, neural interfaces in the future will play a crucial role for design and implementation of prosthetic devices, to replace lost sensory or motor function (Horch and Dhillon, 2004). The success of such systems will depend upon improvement of implant biocompatibility to prevent loss of signal quality over time.

\section{Conclusion}

This work presents implantable, flexible polymer-based microelectrodes for both acute and chronic neural recording in rodents. These recordings yielded similar results, compared to commercially available silicon-based microelectrodes and produced response waveforms of undiminished amplitude and signal-to-noise ratios 8 weeks after a chronic implantation. Preliminary immunostaining results show minimal tissue reaction to the implanted polymer-based device. Current work continues to use these microelectrodes in long-term trials in awake animals.

Implantable flexible microelectrode arrays may in the future contribute to the realization of neuroprosthetic devices to restore nervous system function. By recording signals from the motor cortex, such probes with the addition of on-board electronics and telemetry could be used for the direct control of prosthetic devices for patients of neuromuscular disease, amputation or stroke. The long-term functionality of these devices will depend on controlling the cellular encapsulation, which isolate these probes from the brain. Future work aims to incorporate functional polymer coatings that contain antibiotics or antiinflammatory compounds to reduce the growth of scar tissue in the vicinity of the implanted probes. In addition, fluidic channels incorporated into the probes will enable localized drug delivery adjacent to the electrode sites.

\section{Acknowledgements}

This work was supported by the Healthy Aims project IST2002-1-001837, the Academy of Finland, the Paolo Foundation, the Instrumentarium Science Foundation and the Cultural Foundations of Finland. Microfabrication was done at the Center of MicroNanoTechnology (CMI) at EPFL.

\section{References}

Bai, Q., Wise, K.D., 2001. IEEE Trans. Biomed. Eng. 48, 911-920.

Bartesaghi, R., Gessi, T., 2003. Hippocampus 13, 235-249.

Bragin, A., Hetke, J., Wilson, C.L., Anderson, D.J., Engeljr, J., Buzsaki, G., 2000. J. Neurosci. Methods 98, 77-82.

Brankack, J., Stewart, M., Fox, S., 1993. Brain Res. 615, 310-327.

Buzsaki, G., 2004. Nat. Neurosci. 7, 446-451.

Campbell, P.K., Jones, K.E., Huber, R.J., Horch, K.W., Normann, R.A., 1991. IEEE Trans. Biomed. Eng. 38, 758-768.

Canning, K.J., Leung, L.S., 1997. Hippocampus 7, 643-655.

Canning, K.J., Wu, K.U.N., Peloquin, P., Kloosterman, F., Leung, L.S., 2000. Ann. N.Y. Acad. Sci. 911, 55-72.

Csicsvari, J., Henze, D.A., Jamieson, B., Harris, K.D., Sirota, A., Bartho, P., Wise, K.D., Buzsaki, G., 2003. J. Neurophysiol. 90, 1314-1323.

de Haro, C., Mas, R., Abadal, G., Munoz, J., Perez-Murano, F., Dominguez, C., 2002. Biomaterials 23, 4515-4521. 
Donoghue, J.P., 2002. Nat. Neurosci. 5, 1085-1088.

Edell, D.J., Toi, V.V., Mcneil, V.M., Clark, L.D., 1992. IEEE Trans. Biomed. Eng. 39, 635-643.

Fawcett, J.W., Asher, R.A., 1999. Brain Res. Bull. 49, 377-391.

Freeman, J.A., Nicholson, C., 1975. J. Neurophysiol. 38, 369-382.

Ges, I.A., Ivanov, B.L., Schaffer, D.K., Lima, E.A., Werdich, A.A., Baudenbacher, F.J., 2005. Biosens. Bioelectron. 21, 248-256.

Gnatkovsky, V., de Curtis, M., 2006. J. Neurosci. 26, 873-881.

Hampton, D.W., Rhodes, K.E., Zhao, C., Franklin, R.J.M., Fawcett, J.W., 2004. Neuroscience 127, 813-820.

Horch, K.W., Dhillon, G. (Eds.), 2004. Neuroprosthetics: Theory and Practice. World Scientific Publishing Company, River Edge, NJ.

Hung, M.W.A., 2003. Micromachined High-Density Electrodes for Retinal Prosthesis. Electrical Engineering. University of California Los Angeles, Los Angeles.

Kloosterman, F., Peloquin, P., Leung, L.S., 2001. J. Neurophysiol. 86, $2435-2444$

Kloosterman, F., Haeften, T.V., Silva, F.H.L.D., 2004. Hippocampus 14, $1026-1039$.

Lemon, R., 1973. In: Phillips, M.I. (Ed.), Brain Unit Activity During Behavior. Thomas, Springfield, Il.

Leung, S., 1979. J. Neurophysiol. 42, 1571-1589.

Leung, L.S., 1990. In: Boulton, A.A., Baker, G.B., Vanderwolf, C.H. (Eds.), Neurophysiological Techniques: Applications to Neural Systems. Humana Press, Clifton, NJ.

Leung, L.S., Shen, B., Kaibara, T., 1992. Neuroscience 48, 63-74.

Leung, L.S., Roth, L., Canning, K.J., 1995. J. Neurophysiol. 73, 2392-2403.

Liu, P., Bilkey, D.K., 1997a. Hippocampus 7, 389-396.

Liu, P., Bilkey, D.K., 1997b. Hippocampus 7, 296-306.

Metz, S., Jiguet, S., Bertsch, A., Renaud, P., 2004a. Lab on a Chip 4, 114120.

Metz, S., Trautmann, C., Bertsch, A., Renaud, P., 2004b. J. Micromech. Microeng. 14, 324-331.

Metz, S., Bertsch, A., Renaud, P., 2005. J. Microelectromech. Syst. 14, 383-391.

Mitzdorf, U., 1985. Physiol. Rev. 65, 37-100.

Nicholson, C., Freeman, J.A., 1975. J. Neurophysiol. 38, 356-368.

Nicolelis, M.A.L., 2001. Nature 409, 403-407.
Nicolelis, M.A.L., Dimitrov, D., Carmena, J.M., Crist, R., Lehew, G., Kralik, J.D., Wise, S.P., 2003. PNAS 100, 11041-11046.

Norton, W.T., Aquino, D.A., Hozumi, I., Chiu, F.-C., Brosnan, C.F., 1992. Neurochem. Res. (Hist. Arch.) 17, 877-885.

Richardson Jr., R.R., Miller, J.A., Reichert, W.M., 1993. Biomaterials 14 627-635.

Rodriguez, F.J., Ceballos, D., Schuttler, M., Valero, A., Valderrama, E., Stieglitz, T., Navarro, X., 2000. J. Neurosci. Methods 98, 105-118.

Rousche, P.J., Pellinen, D.S., Pivin Jr., D.P., Williams, J.C., Vetter, R.J., Kirke, D.R., 2001. IEEE Trans. Biomed. Eng. 48, 361-371.

Schmidt, S., Horch, K., Normann, R., 1993. J. Biomed. Mater. Res. 27, 1393-1399.

Seo, J.-M., Kim, S.J., Chung, H., Kim, E.T., Yu, H.G., Yu, Y.S., 2004. Mater. Sci. Eng.: C 24, 185-189.

Shehab, S.A.S., Cronly-Dillon, J.R., Nona, S.N., Stafford, C.A., 1990. Brain Res. 518, 347-352.

Stieglitz, T., Beutel, H., Schuettler, M., Meyer, J.-U., 2000. Biomed. Microdevices 2, 283-294.

Stieglitz, T., Ruf, H.H., Gross, M., Schuettler, M., Meyer, J.-U., 2002. Biosens. Bioelectron. 17, 685-696.

Strumwasser, F., 1958. Science 127, 469-470.

Subbaroyan, J., Martin, D.C., Kipke, D.R., 2005. J. Neural Eng. 2, 103.

Szarowski, D.H., Andersen, M.D., Retterer, S., Spence, A.J., Isaacson, M., Craighead, H.G., Turner, J.N., Shain, W., 2003. Brain Res. 983, 23-35.

Taylor, D.M., Tillery, S.I.H., Schwartz, A.B., 2002. Science 296, 1829-1832.

Townsend, G., Peloquin, P., Kloosterman, F., Hetke, J.F., Leung, L.S., 2002. Brain Res. Protoc. 9, 122-129.

Turner, J.N., Shain, W., Szarowski, D.H., Andersen, M., Martins, S., Isaacson, M., Craighead, H., 1999. Exp. Neurol. 156, 33-49.

Van Hoesen, G.W., 1982. Trends Neurosci. 5, 345-350.

Wise, K.D., Anderson, D.J., Hetke, J.F., Kipke, D.R., Najafi, K., 2004. Proc. IEEE 92, 76-97.

Witter, M., 1993. Hippocampus 3, 33-44.

Wu, K., Leung, L.S., 2001. Neuroscience 104, 379-396.

Wu, K., Leung, L.S., 2003. Neuroscience 116, 599-616.

Zhong, Y., Yu, X., Gilbert, R., Bellamkonda, R.V., 2001. J. Rehabil. Res. Dev. $38,627-632$. 